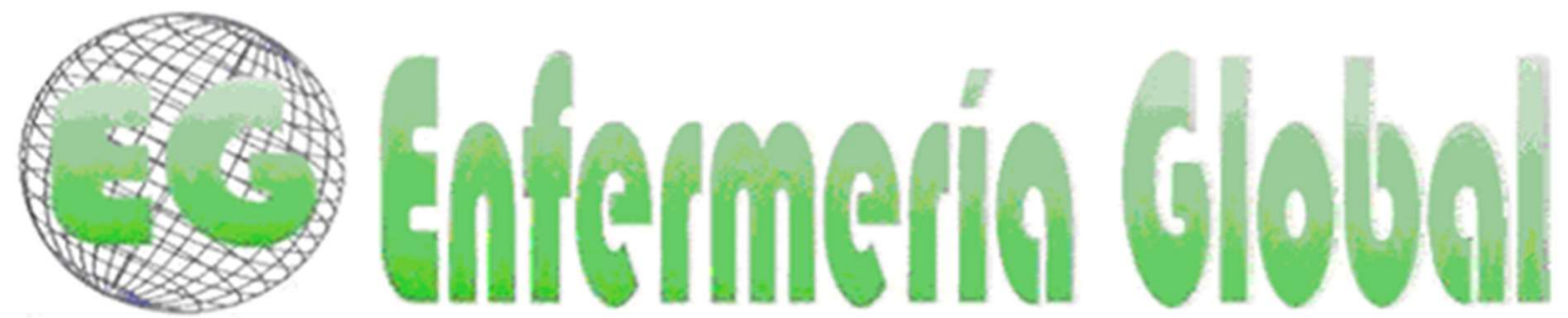

\title{
ORIGINALES
}

\section{Diseño y validación de un video educativo para la prevención del VPH}

Design and validation of an educational video for HPV prevention

Francisco Javier Báez ${ }^{1}$

Félix Chang-Chong ${ }^{2}$

María José Ortiz Hernández ${ }^{1}$

Vianet Nava Navarro ${ }^{1}$

Miguel Angel Zenteno López ${ }^{1}$

Marcela Flores Merlo ${ }^{1}$

1 Facultad de Enfermería Benemérita Universidad Autónoma de Puebla. México. javier.baez@correo.buap.mx

2 Facultad de Medicina. Universidad Autónoma de Chiapas, México.

\section{https://doi.org/10.6018/eglobal.445631}

Recibido: 24/09/2020

Aceptado: $13 / 01 / 2021$

\section{RESUMEN:}

Objetivo: Diseñar y validar un video educativo para la prevención del VPH en jóvenes, mediante el modelo de información, motivación y habilidades conductuales.

Metodología: El diseño del presente estudio consistió en seis etapas: 1.- revisión descriptiva de literatura; 2.- análisis de entrevistas con la población blanco; 3.- ubicación de la información obtenida (literatura y entrevistas), en los componentes del modelo de información - motivación - habilidades conductuales (IMH); 4.- elaboración de guión, 5.- proceso de validación de expertos y 6.- prueba piloto.

Resultados: Con base en los pasos anteriores, se diseño el video titulado: " $i$ cosas que debes saber sobre el VPH!", donde se presentan dos jóvenes (hombre y mujer), que responden de forma clara y sencilla siete preguntas sobre el VPH, finalizando con una serie de recomendaciones para prevenir la infección y promover una sexualidad responsable. La validación se realizó mediante un grupo focal de 10 jóvenes, reunidos en una plataforma en línea y mediante el índice de validez de contenido (IVC), obteniendo un valor de .92, considerado como bueno y adecuado para comprender los aspectos básicos acerca del VPH.

Conclusiones: El diseño y validación de un video para la prevención del VPH es un proceso metodológico hermenéutico y sistemático, que promueve el pensamiento ecléctico, heurístico e innovador para la prevención y promoción de una sexualidad responsable en la población joven.

Palabras-clave: Estudio de Validación; Recursos Audiovisuales; Infecciones por Papillomavirus.

\section{ABSTRACT:}

Objective: To design and validate an educational video for the prevention of HPV in young people, using the information, motivation and behavioral skills model. 
Methodology: The design of the present study consisted of six stages: 1.- descriptive literature review; 2.- analysis of interviews with the target population; 3.- placing the information obtained (literature and interviews) within the components of the information - motivation - behavioral skills model (IMB); 4.script development, 5.- expert validation process, and 6.- pilot test.

Results: Based on the previous steps, the video titled: "7 things you should know about HPV!" was designed, where two young people (a man and a woman) appear and answer, in a clear and simple way, seven questions about HPV, ending with a series of recommendations to prevent infection and promote responsible sexuality. Validation was carried out using a focus group of 10 young people gathered in an online platform and the content validity index (CVI), obtaining a value of .92, which is considered good and adequate to understand the basic aspects of HPV.

Conclusions: The design and validation of a video for the prevention of HPV is a hermeneutical and systematic methodological process that promotes eclectic, heuristic, and innovative thinking for prevention and promotion of responsible sexuality in the young population.

Keywords: Validation Study; Audiovisual resources; Papillomavirus Infections.

\section{INTRODUCCIÓN}

La infección por el virus del papiloma humano (VPH), es el principal responsable del cáncer cervicouterino y otros tipos de cánceres, así como de verrugas anogenitales en varones y mujeres de mayor incidencia en el mundo, en poblaciones sexualmente activas, con un mayor índice de contagio en jóvenes entre los 15 y 25 años de edad(1).

A nivel mundial, se estima que la prevalencia de VPH en mujeres es de $11.7 \%$, mientras que en hombres es alta en todas las regiones del mundo $(21 \%)$ y el pico suele ocurrir un poco más tarde que en las mujeres (2). En México, en la última semana del año 2019 y primera del 2020, hubo un total de 24,055 casos, de los cuales 22,536 pertenecen a mujeres y 1,519 a hombres, a su vez, Puebla y Chiapas se ubicaron dentro de los primeros quince estados a nivel nacional con mayor número de personas infectadas, con un total de 1,264 (3).

Entre los principales factores de riesgo para desarrollar una infección de VPH, se encuentra el tabaquismo (generalmente en las mujeres), las relaciones sexuales tempranas sin protección (vaginal, oral, anal y/o de cualquier otro tipo de contacto en la región genital), el número de parejas sexuales, mujeres con alto número de embarazos, el parto en edades tempranas, la depresión del sistema inmunológico, el uso prolongado de anticonceptivos y la mala nutrición, así como una falta de conocimiento acerca de esta infección ${ }^{(4)}$.

En este sentido, estudios recientes realizados en Turquía, Brasil, México y Estados Unidos, coinciden que el mayor desconocimiento en la población es con referencia a: ¿qué es el VPH?, ¿quién es el portador del virus?, la aplicación de vacunas en ambos géneros, así como de los serotipos más comunes ${ }^{(4-9)}$. Resultados, que se relacionan a conductas sexuales de riesgo, como el coito vaginal y/o anal sin protección, las múltiples parejas sexuales y el uso de drogas durante las relaciones sexuales ${ }^{(10,11)}$.

Con base en lo anterior, se hace evidente el hecho de que los conocimientos sobre esta infección juegan un papel importante sobre la forma en que las personas podrían asumir la responsabilidad de su vida sexual. Es por ello que diversas investigaciones han demostrado que fomentar en jóvenes y adolescentes el conocimiento acerca del VPH, tanto en la promoción y prevención, a través de recursos audiovisuales educativos (videos), que reflejen situaciones reales, en la que un grupo de jóvenes aprenden a reducir el riesgo de padecer infecciones de transmisión sexual (ITS), 
resultan favorables para modificar: mitos, creencias y formas de conductas que hayan influido en su formación y así contribuir a que en su etapa de adultos manifiesten autorresponsabilidad en el cuidado de su salud sexual (12), mediante el desarrollo de habilidades conductuales especificas como la realización de pruebas de detección de $\mathrm{VPH}$, uso y negociación del condón, así como el convencimiento de la vacunación en grupos vulnerables ${ }^{(13-15)}$.

Es aquí, que resulta relevante el diseño y validación de videos educativos con una base teórica, que permita comprender y provocar cambios en el comportamiento de la salud. Motivo, por el cual, el modelo de información, motivación y habilidades conductuales (IMH) de Fisher y Fisher ${ }^{(16)}$, es utilizado en el presente estudio, debido a su propuesta de que la adopción de una conducta preventiva, requiere aumentar el conocimiento, la motivación y las habilidades relacionadas con dicho comportamiento. Indica que la ganancia de información es un precursor necesario para adoptar una conducta preventiva, y que la motivación para cambiar es más importante si se aprende cómo ejecutar dicha conducta. El modelo establece que las habilidades conductuales relacionadas con acciones de prevención, son una vía final común de la información y la motivación. La ganancia de habilidades de comportamiento para lograr este cambio se vuelve decisivo cuando las acciones preventivas requieren habilidades complejas, tales como asistir a realizarse pruebas de detección de VPH, uso y negociación del condón con una pareja y la vacunación en grupos vulnerables ${ }^{(17,18)}$.

Por todo lo anterior señalado, se planteó el siguiente objetivo: diseñar y validar un video educativo para la prevención del VPH en jóvenes, mediante el modelo de información, motivación y habilidades conductuales.

\section{METODOLOGÍA}

El diseño del presente estudio consistió en seis etapas: I.- revisión descriptiva de literatura; II.- análisis de entrevistas con la población blanco; III.- ubicación de la información obtenida (literatura y entrevistas) en los componentes del modelo de información - motivación - habilidades conductuales (IMH); IV.- elaboración de guión, V.- proceso de validación de expertos y VI.- prueba piloto.

\section{(I) Revisión descriptiva de literatura}

El primer paso consistió en realizar una búsqueda de literatura acerca del conocimiento sobre el VPH y las principales herramientas educativas utilizadas en la prevención y promoción de la salud sexual en población joven, publicada en los últimos cinco años (2015 al 2020), en idioma español, inglés y portugués. La búsqueda se realizó en bases de datos científicas (EBSCO, PUBMED, SCOPUS, CINAHL, WEB OF SCIENCE) y en el repositorio digital del Consejo Nacional de Ciencia y Tecnología (CONRYCIT), mediante el uso de operadores boléanos (AND, OR, NOT) y palabras clave de los descriptores en ciencias de la salud (DeCS), donde se construyeron cadenas de búsqueda. Además, se elaboró una matriz de análisis de estudios que contenía: objetivo, diseño (población, muestra), resultados, conclusiones y datos de referencia. 


\section{(II) Análisis de entrevistas con la población blanco}

El segundo paso, consistió en realizar 10 entrevistas semiestructuradas a la población blanco (cinco hombres y cinco mujeres de los estados de Puebla y Chiapas), mismas que fueron grabadas y analizadas por el equipo de investigación. Tuvieron como objetivo, 1.- Saber, ¿qué conocimiento tenían del VPH? y 2.- identificar las características que deberían tener los mensajes, así como la duración y forma del video, para aprender sobre el VPH.

\section{(III) Ubicación de la Información obtenida en los componentes del modelo IMH}

El tercer paso fue la ubicación de la información obtenida a través de la literatura científica y las entrevistas realizadas a la población blanco, en los componentes del modelo de información - motivación - habilidades conductuales (IMH) para el diseño preliminar del video. Se tomó la propuesta de Menacho y Blas ${ }^{(17)}$, adaptada por Báez, Márquez, Onofre, Benavides y Flores ${ }^{(19)}$, que consistió en la construcción de una matriz de relación de conceptos del marco teórico, los componentes del video (información y contenido), así como los resultados esperados con base al objetivo que se pretende lograr, a fin de planificar la elaboración de este recurso audiovisual educativo, con la propuesta teórica.

\section{(IV) Elaboración de guión}

El cuarto paso consistió en la elaboración del guión, mediante el trabajo multidisciplinario, integrado por un diseñador grafico, un comunicólogo y un guionista con experiencia en la elaboración de videos relacionados con temas de salud. El equipo de investigación junto con la productora, realizaron el casting, eligieron a los actores (voces), imágenes, música y locaciones para el video.

Para la elaboración del guión se consideraron las sugerencias reportadas por las entrevistas a la población blanco, con mensajes básicos y cortos, mediante un lenguaje e imágenes simples, naturales y con actores jóvenes con personalidades que existen en la vida real. Además, se consideraron las recomendaciones de los estudios relacionados, así como de la información acerca del VPH contenida en la Organización Mundial de la Salud, el Center for Disease Control and Prevention (CDC) y la Secretaría de Salud en México. Lo que permitió mejorar los contenidos de los mensajes en el video.

\section{(V) Proceso de validación de expertos}

El quinto paso fue la validación de los contenidos de la primera versión del video, por parte de 6 expertos en la temática de salud sexual, así como de un grupo focal de 10 jóvenes, reunidos en una plataforma en línea, con el propósito de conocer su opinión general y tener la seguridad de que la información y los mensajes se comprendieron correctamente. Dicho ejercicio, permitió determinar la eliminación y/o edición de alguna escena. 


\section{(VI) Prueba Piloto}

Finalmente, el sexto paso fue la elaboración de una prueba piloto con un grupo de 30 jóvenes (debido a que este video se usará para una intervención en internet), los cuales tuvieron acceso desde una pagina web, para conocer su opinión de ver el video a través de este medio.

Cabe señalar que la información obtenida de las entrevistas y del grupo focal, fue analizada mediante un muestreo por saturación de datos, donde se identificaron conceptos y temas emergentes, para crear categorías y códigos de análisis (20; 21). Asimismo, es importante aclarar que esta investigación se rigió por los principios de Bioética y la Ley General Salud de México, en su apartado de investigación con seres humanos, al contar con consentimiento informado por todos los participantes, así como con número de registro ante un comité de investigación (SIEP/033/2020).

\section{RESULTADOS}

\section{(I) Revisión descriptiva de literatura}

La primera etapa del estudio tuvo como objetivo, identificar las principales características del tema, mediante una revisión descriptiva de literatura. La cual dio como resultado, un alto desconocimiento en jóvenes acerca del VPH, principalmente en: no saber que afecciones pueden generar el virus, la falta de información sobre la vacuna y las pruebas de detección $(7,22)$. Situación que puede contribuir a la mala praxis en su salud sexual, o a tener actividades riesgosas como: el tener múltiples parejas sexuales y no usar el preservativo ante un contacto sexual. (4) Evidencia científica que pone en descubierto la necesidad de realizar intervenciones sobre el $\mathrm{VPH}$, a fin de informar entre otras cosas, las medidas de prevención y los factores de riesgo ${ }^{(6)}$.

En cuanto a las principales herramientas educativas utilizadas en la prevención y promoción de la salud sexual en población joven, se halló que el uso de medios audiovisuales, como son los videos, donde se informa de forma general, aspectos relacionados a la salud sexual, resultan efectivos para mejorar las conductas de prevención de ITS (23).

\section{(II) Análisis de entrevistas con la población blanco}

En la segunda etapa, se entrevistaron 10 jóvenes entre los 18 y 23 años de edad, 2 se encontraban estudiando en el nivel medio superior, otros 2 administración de empresas, mientras que los demás refirieron estudiar las carreras de gastronomía, derecho, nutrición, psicología, multimedia y física.

Del análisis de sus respuestas, emergieron las siguientes categorías, en cuanto al conocimiento y a las características preferidas para aprender sobre el VPH: 1.- La desinformación sobre: signos, síntomas, factores de riesgo y prevención, 2.- tipo de video, 3.- uso de imágenes y 4.- estilo de personajes.

Para la primera categoría, se muestra un desconocimiento claro sobre los signos, síntomas, factores de riesgo y prevención del VPH. 
"...desconozco realmente cuales son los signos y síntomas...creo que con los métodos anticonceptivos se puede prevenir (E3: 20 años)"; "es una enfermedad que generalmente tienen las mujeres, pero a los hombres les ataca más (E2: 21)"; "No sé, si llegan a presentar síntomas, eso si no tengo idea...desconozco si existen vacunas (E5: 19 años)"; "no se, si las relaciones sexuales influyen para tener VPH...considero que un factor, es no usar anticonceptivos (E10: 23 años)".

Respecto al tipo de video, destacó en los participantes aquellos que utilizan información clara, concisa y concreta sobre el VPH, con un tiempo de duración no mayor de cinco minutos.

"Un video rápido... no mas de cinco minutos (E4: 18 años)"; "prefiero un video no aburrido ni tedioso, con información clara, concisa y fundamental (E6: 21 años); "Un video preciso, conciso, didáctico y que no este lleno de letras (E8: 19años)".

En cuanto al uso de imágenes, refirieron en su mayoría, que fueran reales, explicitas y del mismo estilo.

"Considero que una imagen real seria mas entendible el concepto (E7: 20 años)"; "yo creo que las imágenes deberían ser un poco más explícitas... para tener una idea (E2: 23 años)"; "creo que resulta más atractivo que todas las imágenes sean del mismo estilo (E7: 20 años)".

Finalmente, para el estilo de personajes argumentaron que fuera gente común, que fuera inclusivos (hombre y mujer) y contemporáneos. Además de que no manejaran mucho un lenguaje médico.

"...que no sea con mucho lenguaje médico, con lenguaje común... (E1: 18 años)"; “...debería ser un video llamativo, algo que sea de estilo más contemporáneo, que no sea forzado, que sea una manera más entre amigos, con personas reales...(E8: 18 años)".

\section{(III) Ubicación de la Información obtenida en los componentes del modelo IMH}

Una vez obtenida la información de los participantes claves y de la literatura científica, se inició con el tercer paso, que consistió en ubicar todos los datos, en los componentes del modelo de información - motivación - habilidades conductuales $(\mathrm{IMH})$ para el diseño preliminar del video. Donde para el primer constructo se considero el conocimiento sobre qué es el VPH y las formas de infectarse. En la motivación se ubicaron los signos y síntomas, así como los factores de riesgo. Las formas de prevención se ubicaron en el constructo de habilidad (Tabla 1). 
Tabla 1: Relación entre el Modelo de información - motivación - habilidades conductuales (IMH), los componentes del video y los resultados esperados.

\begin{tabular}{|c|c|c|}
\hline $\begin{array}{c}\text { Modelo } \\
\text { I-M-H }\end{array}$ & Contenido del video & Objetivo \\
\hline Información & $\begin{array}{l}\text { “...El 395exua de papiloma humano } \\
\text { también conocido por sus siglas VPH, es } \\
\text { uma de las infecciones de transmisión } \\
\text { sexual más común uma hombres y mujeres } \\
\text { uma vida sexual activa..." }\end{array}$ & $\begin{array}{l}\text { Dar a conocer la } \\
\text { definición y la forma } \\
\text { de infección del VPH }\end{array}$ \\
\hline Motivación & $\begin{array}{l}\text { “...Entre los tipos de VPH de bajo riesgo su } \\
\text { mayor característica es la aparición de } \\
\text { verrugas uma la zona genital, así como } \\
\text { uma el ano, boca o muslos...El principal } \\
\text { 395exua de riesgo es por contacto sexual } \\
\text { sin protección...” }\end{array}$ & $\begin{array}{l}\text { Mostrar las } \\
\text { consecuencias del } \\
\text { VPH, así como los } \\
\text { factores de riesgo } \\
\text { para contraer la } \\
\text { infección }\end{array}$ \\
\hline Habilidad & $\begin{array}{l}\text { "Recuerda que... desde el inicio de tu Vida } \\
\text { Sexual Activa, realízate uma prueba de } \\
\text { Papanicolaou o detección de híbridos para } \\
\text { VPH anualmente, o si cumples uma algún } \\
\text { signo de alarma u otros factores de riesgo. } \\
\text { Recuerda que el VPH no se ve a simple } \\
\text { vista..." }\end{array}$ & $\begin{array}{l}\text { Reforzar uma el } \\
\text { participante las } \\
\text { acciones de } \\
\text { prevención, } \\
\text { detección y } \\
\text { promoción para uma } \\
\text { 395exualidade } \\
\text { saludable. }\end{array}$ \\
\hline
\end{tabular}

Fuente: Elaboración propia de los autores.

\section{(IV) Elaboración de guión}

Con base en lo anterior, para el cuarto paso, se decidió con el equipo multidisciplinario, diseñar el video titulado: "i7 cosas que debes saber sobre el VPH!", donde se presentan dos jóvenes (hombre y mujer); que responden de forma clara y sencilla, siete preguntas sobre el VPH, finalizando con una serie de recomendaciones para prevenir la infección y promover una sexualidad responsable. El video fue elaborado mediante los elementos de la comunicación persuasiva ${ }^{(24)}$, la cual se caracteriza por tratar de producir un cambio positivo en las conductas de salud de las personas mediante: 1.- una fuente atractiva y personajes semejantes al receptor, 2.una organización de los mensajes claros y racionales, 3.- un canal comunicativo visual - auditivo directo y 4.- bajo un contexto relajado y agradable para los receptores. Aspectos que han permitido en estudios anteriores, cambiar creencias e inspirar comportamientos sexuales saludables $(25,26)$.

\section{(V) Proceso de validación de expertos}

Una vez terminado el guión, se procedió con el quinto paso, el cual consistió en la validación de los contenidos de la primera versión del video, por parte de 6 expertos en la temática de salud sexual, así como de un grupo focal de 10 jóvenes, reunidos en una plataforma en línea. Para lo cual, se calculo el índice de validez de contenido (IVC) propuesto por Rubio (27), el cual mencionan que para considerar adecuado el contenido de un instrumento, deberá obtener un valor mínimo de .80 (Tabla 2). 
Tabla 2: Validez de contenido por Jueces.

\begin{tabular}{|l|c|c|c|c|c|c|c|}
\hline \multirow{2}{*}{ Pregunta / Respuestas } & \multicolumn{7}{|c|}{ Expertos } \\
\cline { 2 - 8 } & 1 & 2 & 3 & 4 & 5 & 6 & IVC \\
\hline ¿Qué es VPH? & 1 & 3 & 4 & 2 & 4 & 3 & $4 / 6=.60$ \\
\hline ¿Cómo se transmite? & 4 & 3 & 4 & 3 & 4 & 3 & $6 / 6=1$ \\
\hline ¿Cuáles son los signos y síntomas? & 4 & 3 & 4 & 2 & 3 & 3 & $5 / 6=.83$ \\
\hline ¿Cuáles son los factores de Riesgo? & 3 & 3 & 4 & 3 & 4 & 4 & $6 / 6=1$ \\
\hline ¿Cómo se previene? & 4 & 3 & 4 & 4 & 4 & 4 & $6 / 6=1$ \\
\hline $\begin{array}{l}\text { ¿Quién y cuándo se debe aplicar la } \\
\text { vacuna? }\end{array}$ & 4 & 3 & 4 & 4 & 3 & 4 & $6 / 6=1$ \\
\hline ¿Cómo se diagnostica? & 3 & 3 & 4 & 4 & 3 & 4 & $6 / 6=1$ \\
\hline Tips de promoción & 4 & 3 & 4 & 4 & 4 & 4 & $6 / 6=1$ \\
\hline IVC Total & \multicolumn{7}{|c|}{} \\
\hline
\end{tabular}

Fuente: IVC= Índice de validez de contenido.

Con base en lo anterior y con las sugerencias realizadas por el grupo focal de jóvenes, se decidió realizar ajustes en los contenidos sobre la definición del VPH, los signos y síntomas. Así como mejorar la redacción de los apartados correspondientes a: formas de transmisión, quién y cuándo se debe aplicar la vacuna, así como las formas de diagnostico, obteniendo un IVC= .92.

\section{(VI) Prueba Piloto}

Finalmente, se realizó una prueba piloto a 30 jóvenes universitarios, a fin de conocer su opinión respecto al video, el cual calificaron como muy bueno, entendible y adecuado, para comprender los aspectos básicos acerca del VPH. Consideraron que el tiempo era adecuado, sugirieron cambios en la música a una de tonos mas agudos, a fin de que no fuera un distractor entre los asistentes, asimismo, señalaron cambiar la ubicación de algunas palabras que se presentaban durante el video y ampliar algunas imágenes, con la finalidad de generar un mayor impacto.

\section{DISCUSIÓN}

La presente investigación tuvo como propósito describir el proceso de diseño y validación de un video educativo para la prevención del VPH, mediante el modelo de información, motivación y habilidades conductuales.

Durante la revisión y análisis de literatura, se encontró una fuerte necesidad de construir herramientas didácticas, para aumentar el conocimiento de prevención para el VPH. Además de coincidir con lo reportado en los estudios relacionados, acerca de la falta de información sobre los signos, síntomas, factores de riesgo y formas de prevención, hallados durante las entrevistas con la población joven. 
Asimismo, se aprecia a través de la operacionalización del modelo de información, motivación y habilidades conductuales, en los componentes del video, que este lente teórico puede ser una herramienta eficaz para dirigir y explicar los procesos de adopción de conductas preventivas, que sumado a las técnicas de la comunicación persuasiva, pudieran generar un efecto significativo en los jóvenes, al promover estrategias de prevención y promoción para una sexualidad responsable, mediante personajes que utilizan el mismo lenguaje y que son de la misma edad. Hecho, que permite una fácil identificación y asimilación de los mensajes en los receptores. Resultado que concuerda con lo referido por Menacho y Blas (17), al referir la importancia de incorporar en el proceso de intervención, a la población objetivo, situación que consolida su propuesta metodológica, en la construcción y diseño de videos educativos para promover la salud sexual.

Finalmente, se debe considerar que los gustos de los jóvenes son cambiantes y totalmente dinámicos, acotados a la cultura en la que se encuentran inmersos, sumado, a que año con año el conocimiento científico avanza en relación a la temática del VPH; hace evidente un área de oportunidad, para continuar con la revisión y en su caso, la actualización de los contenidos encontrados en las herramientas audiovisuales educativas (videos), a fin de hacerlas pertinentes entre los jóvenes.

\section{CONCLUSIONES}

El diseño y validación de un video para la prevención del VPH es un proceso metodológico hermenéutico y sistemático, que promueve el pensamiento ecléctico, heurístico e innovador para la prevención y promoción de una sexualidad responsable en la población joven.

El contenido de la presente investigación, pretende ser un referente para la construcción de videos educativos, dirigidos a la promoción de la salud. Liderado por profesionales de enfermería preocupados en la salud sexual.

El presente estudio contribuye a promover un dialogo continuo con la población blanco y la literatura científica, así como un acercamiento al trabajo multidisciplinario y participativo, que permita una mejor reflexión para resolver de manera creativa e innovadora, las necesidades de salud de las poblaciones.

\section{REFERENCIAS}

1. Organización Panamericana de la Salud [Internet]. Acerca del VPH. El virus. [Obtenido: el 11 de agosto de 2020]. Disponible en: https://www.paho.org/hq/index.php?option=com_content\&view=article\&id=1471 8:about-hpvvaccine\&ltemid=72405\&lang=es\#: :text=La\%20prevalencia\%20del\%20VPH\%2 Oen,en\%20la\%20regi\%C3\%B3n\%20del\%20perineo.

2. Organización Mundial de la Salud [Internet]. Acerca del VPH. Carga de la Enfermedad. [Obtenido el 10 de septiembre de 2020]. Disponible en: https://www.paho.org/hq/index.php?option=com_content\&view=article\&id=1471 8:about-hpv- 
vaccine\&ltemid=72405\&lang=es\#: :text=La\%20prevalencia\%20del\%20VPH\%2 Oen,en\%20la\%20regi\%C3\%B3n\%20del\%20perineo

3. Dirección General de Epidemiología [Internet]. Boletín Epidemiológico. Sistema Nacional de Vigilancia Epidemiológica. Sistema Único de Información. Semana 31. [obtenido el 11 de agosto de 2020]. Disponible en: https://www.gob.mx/salud/documentos/boletinepidemiologico-sistema-nacionalde-vigilancia-epidemiologica-sistema-unico-de-informacion-231750

4. Contreras-González R, Magaly-Santana A, Jiménez-Torres E, Gallegos-Torres $\mathrm{R}$, Xeque-Morales Á, Palomé-Vega $\mathrm{G}$, et al. Nivel de conocimientos en adolescentes sobre el virus del papiloma humano. Enfermería Universitaria [Internet], 2017 [obtenido el 10 de septiembre de 2020]; 14 (2): 104-110. Disponible en: http://dx.doi.org/10.1016/j.reu.2017.01.002

5. Dönmez S, Öztürk R, Kısa S, Karaoz-Weller B, Zeyneloğlu S. Knowledge and perception of female nursing students about human papillomavirus (HPV), cervical cancer, and attitudes toward HPV vaccination. Journal of American College Health, [Internet]. 2019 [obtenido el 10 de septiembre de 2020]; 67 (5): 410-417. Disponible en: DOI: 10.1080 / 07448481.2018.1484364

6. Martínez-Martínez L, Cambra U. Conocimiento y actitudes hacia el virus del papiloma humano en una población de universitarios españoles $=$ Knowledge and attitudes towards human papillomavirus in a population of Spanish university students. Revista española de comunicación en salud, [Internet] 2018 [obtenido el 10 de septiembre de 2020]; 9(1): 14-21. Disponible en: https://doi.org/10.20318/recs.2018.4248

7. Hernández-Márquez C, Brito-García I, Mendoza-Martínez M, Yunes-Díaz E, Hernández-Márquez E. Conocimiento y creencias de mujeres del estado de Morelos sobre el virus del papiloma humano. Rev. Cuba Enf. [Internet] 2016 [obtenido el 10 de septiembre de 2020]; 32(4):126-147. Disponible en: http://scielo.sld.cu/pdf/enf/v32n4/enf04416.pdf

8. Galbraith-Gyan K, Lechuga J, Jenerette C, Palmer M, Moore A, Hamilton J. African-American parents' and daughters' beliefs about HPV infection and the HPV vaccine. Public Health Nursing. [Internet] 2019 [obtenido el 10 de septiembre de 2020]; 36:134 - 143. Disponible en: https://doi.org/10.1111/phn.12565

9. Pinheiro $\mathrm{P}$, Miranda Cadete M. El conocimiento de los adolescentes escolarizados sobre el virus del papiloma humano: revisión integrativa. Enfermería Global, [Internet] 2019 [obtenido el 10 de septiembre de 2020]; (56): 603-623. Disponible en: http://dx.doi.org/10.6018/eglobal.18.4.362881

10. Barrios-Puerta Z, Díaz-Pérez A, Del Toro-Rubio M. Conocimientos acerca del Virus de Papiloma Humano y su relación con la práctica sexual en estudiantes de Ciencias de la Salud en Cartagena-Colombia. Ciencia y Salud Virtual. [Internet] 2016 [obtenido el 10 de septiembre de 2020]; 8(1): 20-28. Disponible en: https://revistas.curn.edu.co/index.php/cienciaysalud/article/view/670/530

11. Soto-Zamalloa C. Relación entre el nivel de conocimiento y prácticas de prevención sobre el virus papiloma humano en gestantes atendidas en el Hospital San Juan de Lurigancho, 2018. [Internet] 2019 [obtenido el 10 de septiembre de 2020]; Disponible en: https://hdl.handle.net/20.500.12672/10433

12. Von Sneidern E, Quijano L, Paredes M, Obando E. Estrategias educativas para la prevención de enfermedades de transmisión sexual en adolescentes. Revista Medica Sanitas. [Internet] 2016 [obtenido el 10 de septiembre de 2020]; 19

(4): 198-207. Disponible en: 
https://www.unisanitas.edu.co/Revista/61/RevTema_Estrategias_educativas.pd $f$

13. Al-Shaikh G, Syed S, Fayed A, Al-Shaikh R, Al-Mussaed E, Khan F, et al. Effectiveness of health education programme: Level of knowledge about prevention of cervical cancer among Saudi female healthcare students. Journal of the Pakistan Medical Association. 2017; 67 (4): 513-520.

14. Lemos M, Rothes A, Oliveira F, Soares L. Raising cervical cancer awareness: Analysing the incremental efficacy of Short Message Service. Health Education Journal. 2017; 76(8): 956-970.

15. Allen W. Increasing Knowledge of Preventing Sexually Transmitted Infections in Adult College Students through Video Education: An Evidenced-based Approach. ABNF Journal. 2017; 28(3): 64-68.

16. Fisher J, Fisher W. Changing AIDS-Risk Behavior. Psychological Bulletin, 1992; 3(3): 455-474.

17. Menacho L, Blas M. ¿Cómo producir un video para promover la prueba del VIH en hombres que tienen sexo con hombres? Rev. Perú Med Exp Salud Publica. 2015; 32 (3): 519-525.

18. Pérez G, Cruess D, Strauss M. A brief information-motivation-behavioral skills intervention to promote human papillomavirus vaccination among college-aged women. Psychol Res Behav Manag. [Internet] 2016 [obtenido el 10 de septiembre de 2020]; 9:285-296. Disponible en: https://doi.org/10.2147/PRBM.S112504

19. Báez $H$, Márquez V, Onofre R, Benavides $T$, Flores $M$. Diseño de una intervención hacia la intensión y uso del condón en HSH. Sinapsis de la psicología en su profesionalización e investigación para la comunidad. Colegio de Psicología del Estado de Nuevo León. [Internet] 2018 [obtenido el 10 de septiembre de 2020]; 170-179. Disponible en: https://www.researchgate.net/publication/325094777_Sinapsis_de_la_Psicologi a_en_su_Profesionalizacion_e_Investigacion_para_la_Comunidad

20. Padgett D. Qualitative methods in social work research: challenges and rewards. 2nd ed. Los Ángeles, California: Sage Publications. 2008.

21. Glaser B, Strauss AL. The Discovery of Grounded Theory: Strategies for Qualitative Research. Chicago: Aldine Transaction. 1967.

22. Bendezu-Quispe G, Soriano-Moreno AN, Urrunaga-Pastor D, VenegasRodríguez G, Benites-Zapata VA. (2020). Asociación entre conocimientos acerca del cáncer de cuello uterino y realizarse una prueba de Papanicolaou en mujeres peruanas. Revista Peruana de Medicina Experimental y Salud Publica. [Internet] 2020 [obtenido el 10 de septiembre de 2020]; 37(1), 17-24. Disponible en: https://dx.doi.org/10.17843/rpmesp.2020.371.4730

23. Nuramalia N, Maria IL, Jafar N. The Effectiveness of Audio-Visual Media Intervention Aku Bangga Aku Tahu (ABAT) toward Adolescent Attitude as a Practice of Prevention of HIV and AIDS Transmission. International Journal of Multicultural and Multireligious Understanding. 2019; 6 (5): 713-719.

24. Moya M. Persuasión y cambio de actitudes. En J. F. Morales (Ed.). Psicología social. Madrid: McGraw-Hill. 2000.

25. Bunn C, Kalinga C, Mtema O, Abdulla S, Dillip A, Lwanda J, et al. Arts-based approaches to promoting health in sub-Saharan Africa: a scoping review. BMJ Global Health. 2020; 5 (5): e001987.

26. Donné L, Hoeks J, Jansen C. Using a narrative to spark safer sex communication. Health education journal. [Internet] 2017 [obtenido el 10 de 
septiembre de 2020]; 76(6): 635-647. Disponible en: https://doi.org/10.1177/0017896917710967

27. Rubio DM, Berg-Weber M, Tebb SS, Lee ES, Rauch S. Objectifying content validity: Conducting a content validity study in social work research. Social Work Research. 2003; 27(2), 94-104. 